\title{
The Application of Retrograde Intrarenal Surgery to Remove a Single Large Kidney Stone During Pregnancy
}

Kantima Jongjitaree

Tawatchai

Taweemonkongsap

Sunai Leewansangtong

Sittiporn Srinualnad

Ekkarin Chotikawanich

Division of Urology, Department of Surgery, Faculty of Medicine Siriraj Hospital, Mahidol University, Bangkok, Thailand
This article was published in the following Dove Press journal: Research and Reports in Urology

Purpose: To report the application and outcome of retrograde intrarenal surgery (RIRS) to remove a large kidney stone during pregnancy.

Patient and Methods: A 30-year-old woman presented with an infected kidney stone $(3 \mathrm{~cm}$ in size) at 4 weeks of pregnancy. We decided to remove the stone due to the possibility of obstruction and infection and chose to carry out this procedure by RIRS. In order to avoid complications associated with anesthetic, the surgery was carried out after the infection had cleared and when the patient had entered the second trimester of pregnancy. First, we used an ureteral access sheath and semi-rigid ureteroscopy to evaluate the ureteral lumen. We confirmed that the ureteral access sheath had been positioned appropriately by direct visualization with a flexible ureterorenoscope. The procedure was then carried out with a radiation-free protocol and without fluoroscopy. Ho-YAG laser lithotripsy was used to fragment the stones, and these fragments were then removed in a stone basket. The patient required three sessions of RIRS to remove the stone in its entirety; during this time, the patient was 18-29 weeks into her pregnancy. During each session, we removed approximately $30 \%$ of the stone. The patient developed fever after the first operation but responded fully to antibiotics. There were no perioperative complications, and the patient only remained in hospital for 3 days. The male infant was delivered by caesarian section at 37 weeks of pregnancy without any health complications.

Results and Conclusion: We successfully removed a large kidney stone from a pregnant patient using an ureteral access sheath and RIRS without fluoroscopy. There were no complications indicating that this procedure can be carried out safely during pregnancy.

Keywords: kidney calculi, pregnancy, retrograde intrarenal surgery, ureteroscopy

\section{Introduction and Background}

Kidney stones occur are known to occur in $10 \%$ of the population at some point during their lifetime. The incidence of kidney stones is 1 in 200-500 pregnancies and while there is no significant difference between pregnant and non-pregnant women with regards to incidence, pregnant women do tend to have more symptoms. ${ }^{1}$ The first option to treat kidney stones during pregnancy is by conservative treatment. ${ }^{1}$ Definite treatment is mostly deferred until delivery. However, certain situations arise in certain patients that necessitate the need to remove kidney stones during pregnancy.

\section{Case Presentation}

A 30-year-old woman presented with fever and pain in her left flank. Initially, she was diagnosed with acute left pyelonephritis. Urine culture identified the presence 
of Escherichia Coli with extended-spectrum betalactamases (ESBL) resistance. At this point in time, the patient did not know that she was pregnant; her last menstrual period (LMP) LMP had occurred approximately 4 weeks previously. Initial evaluation by KUB films revealed a left kidney stone that was approximately $3 \mathrm{~cm}$ in diameter (Figure 1A). Ultrasonography of the urinary system showed moderate hydronephrosis of the left kidney along with a left renal pelvic stone that was approximately $3 \mathrm{~cm}$ in diameter (Figure 1B). Ultrasonography of the bladder revealed there was a gestational sac in the uterus (Figure 1C). Consideration of her LMP indicated that the patient was 4 weeks into pregnancy. Therefore, the final diagnosis was an infected left kidney stone (3 $\mathrm{cm}$ in diameter) during pregnancy. We had two possible plans: to remove the stone on the basis of obstruction and infection, or diversion alone. Following discussion between the obstetrician and the patient, we decided upon active management. We chose to carry out retrograde intrarenal surgery (RIRS) in different stages.

To avoid complications associated with the anesthetic, the patient was scheduled for surgery once the infection had cleared and she had entered the second trimester of pregnancy. At the time of surgery, preoperative urine culture was sterile. However, intravenous antibiotic prophylaxis (Meropenem; dose in accordance with the sensitivity of the first infection) was administered prior to the procedure. The procedure was performed by an experienced urologist who had carried out over 1000 cases involving flexible ureteroscopy. The procedure began with semi-rigid ureteroscopy to evaluate the ureteral lumen. An ureteral access sheath (UAS; $11 / 13 \mathrm{Fr}$ ) was selected and positioned without force and using the stiff guidewire. We estimated the length of UAS required to reach the upper ureter. This procedure was successfully completed using a non-pre-stented ureter. We then confirmed that the UAS was positioned appropriately by direct visualization with a flexible ureterorenoscope. The procedure was then carried out with a radiation-free protocol without fluoroscopy. We also ensured that ultrasound was available in the operating theatre if needed.

The stone was broken into fragments with a Ho-YAG laser lithotripsy (Versapulse, Lumenis ${ }^{\circledR}$ ) and the fragments were removed in a basket; the laser was set to 1 Joule and 20 Hertz. During the procedure, we used the lowest possible irrigation flow to achieve an acceptable level vision (Figure 2 ). Due to concerns relating urosepsis, ${ }^{2}$ we restricted the operative time to 60 minutes. The residual stone was left in situ for the second stage of surgery. An ureteral stent (6Fr) was inserted after surgery owing to the presence of the residual stone, a history of urinary tract infection, the application of a UAS, and the need to reduce postoperative pain in high-risk groups. ${ }^{3}$ Although the surgery was performed safely in accordance with strict guidelines, the patient developed postoperative fever but responded fully to antibiotics (Meropenem). Subsequent stone cultures were positive for E. coli, as seen previously with urine cultures from the first infection. In total, three sessions of RIRS were required to

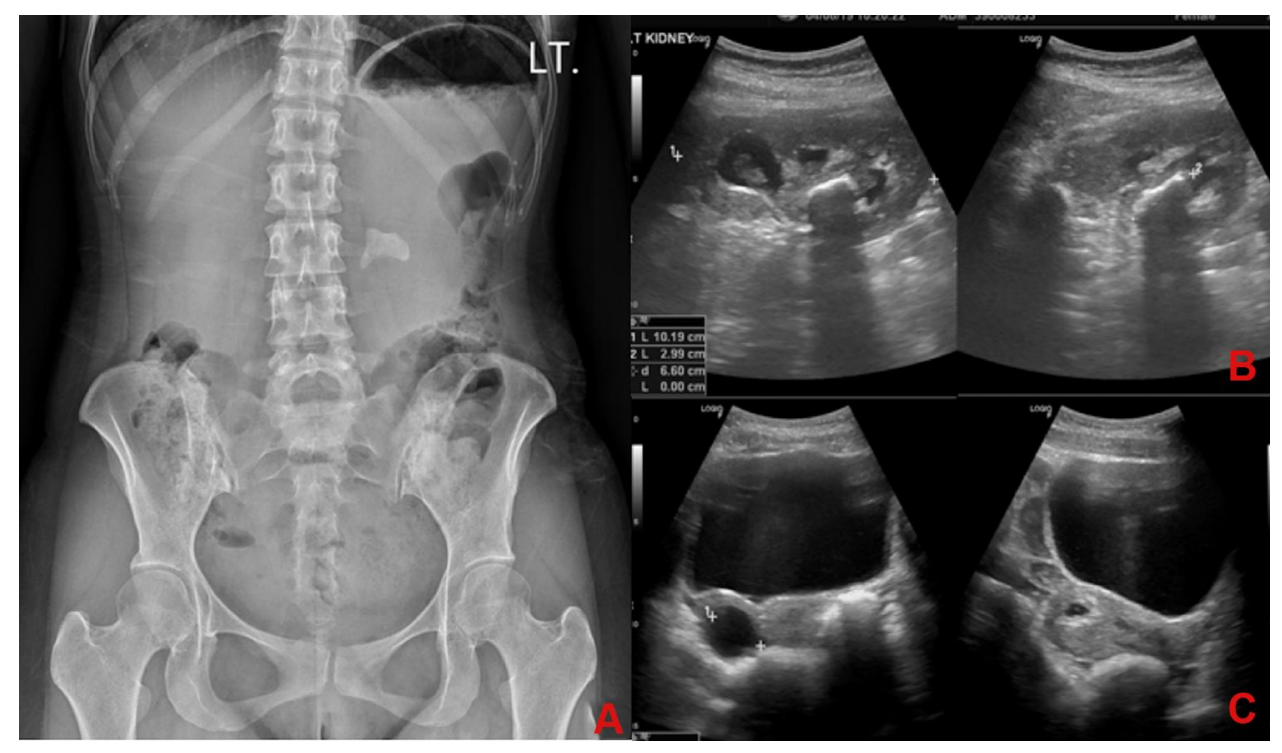

Figure I (A) KUB films at a gestational age of 4 weeks showing a left kidney stone that was approximately $3 \mathrm{~cm}$ in diameter. (B) Ultrasonography of the left kidney showing left hydronephrosis and a left kidney stone. (C) Ultrasonography of the bladder showing a normal bladder mucosa and a gestational sac in the uterus. 


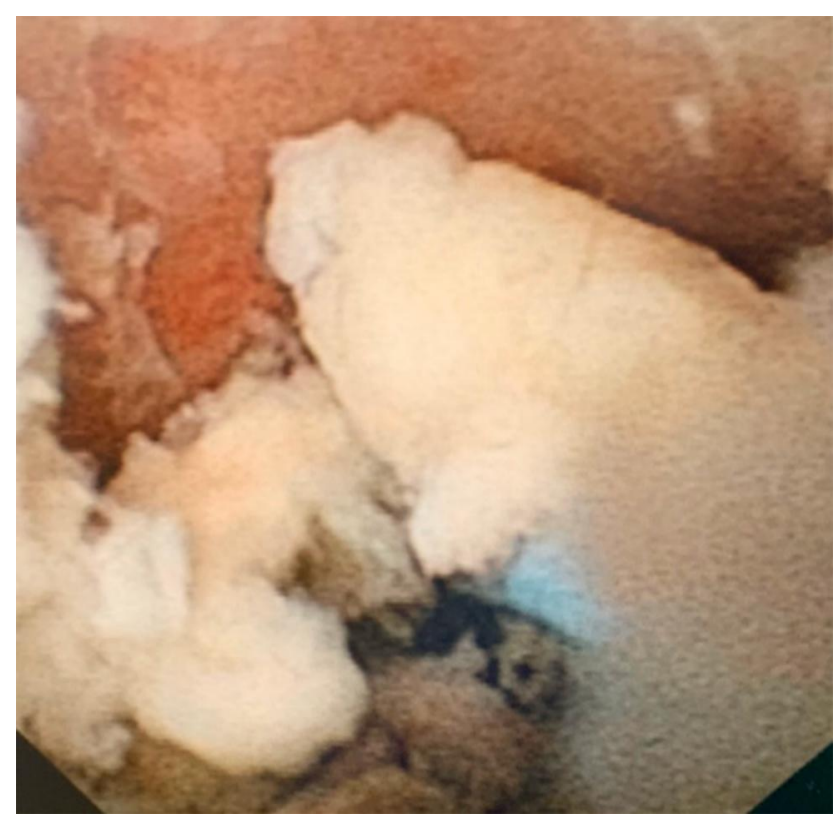

Figure 2 Endoscopic findings related to the infected kidney stone.

fully remove the stone; these operations took place while the patient was 18-29 weeks pregnant (Figure 3). We removed approximately $30 \%$ of the stone during each session of RIRS; these restrictions were caused by issues related to time and irrigation flow. In the second and third operations, we used a larger UAS (12/14 Fr), without insertion difficulty or ureteric injury. There were no perioperative complications after the second and third procedures. The patient remained in hospital for a total of 3 days. The fetus was monitored by an obstetrician at each stage of the surgery and was in good health both before and after the surgical intervention. The ureteral stent was removed 2 weeks after the final procedure. During the remainder of the pregnancy, we used ultrasound to monitor for stones and hydronephrosis; there were no residual stones and no evidence of hydronephrosis when evaluated 2 and 6 weeks after the final surgery.

Nine weeks after the final surgery (at a gestational age of 37 weeks), the patient developed hypertension without proteinuria. She was subsequently diagnosed with pre-eclampsia without severe features. The pregnancy was then terminated by caesarian section and a male infant was delivered without complications. Four months after the final surgery, we used KUB films to check that the patient remained stone-free (Figure 4). Analysis of the stone indicated the presence of calcium phosphate (major) with calcium oxalate monohydrate.

\section{Discussion}

Most kidney stones during pregnancy are managed by conservative treatment. However, the failure to achieve spontaneous passage creates a significant dilemma with regards to treatment. Previous literature proposed that ureteric calculi should be removed by semi-rigid ureteroscopy. ${ }^{1,3}$ However, there is still debate surrounding the circumstances that lead to the need to actively remove kidney stones.

In this report, we describe a pregnant patient with a kidney stone measuring $3 \mathrm{~cm}$ in diameter; the possibility of spontaneous passage was likely to be nil. Active stone management was considered to avoid obstetric complications such as abortion, premature rupture of the membrane, and preterm labor arising from urinary tract infection. Considering the possibility of infection and obstruction

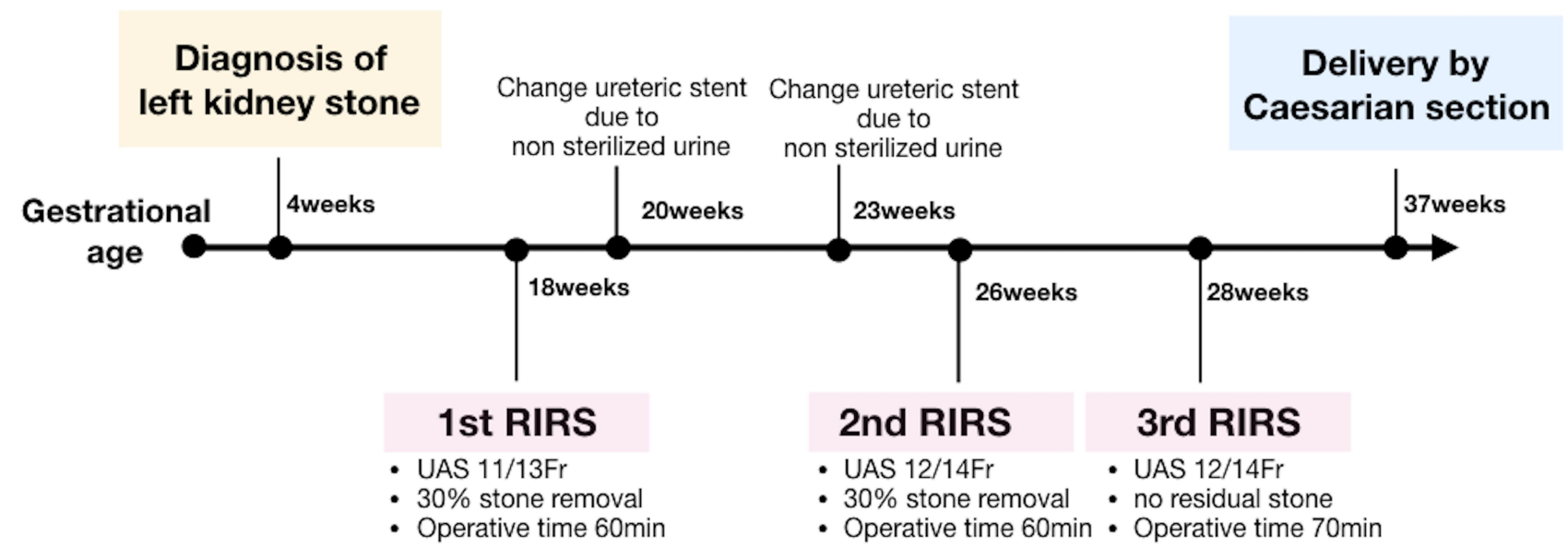

Figure 3 Timeline of kidney stone management. 


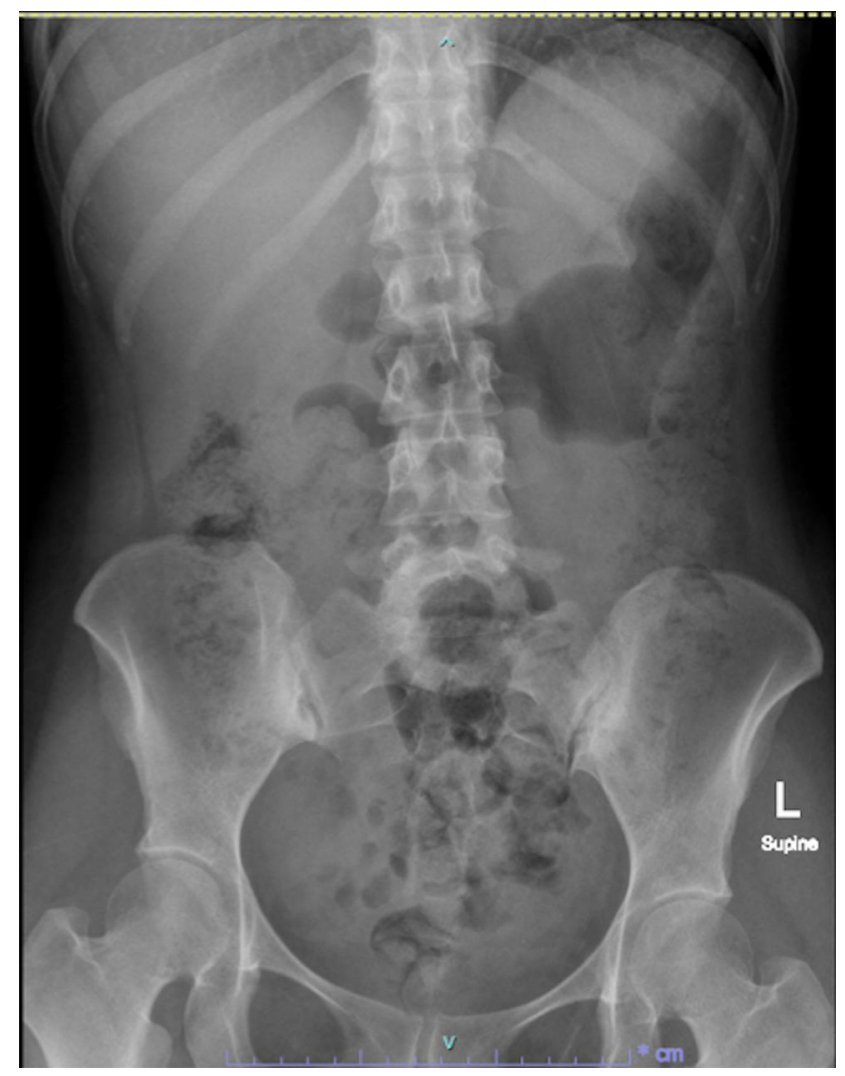

Figure 4 KUB films acquired 4 months after the final surgery.

in our patient, we had two options: diversion alone, or the active removal of the stone. This was a significant dilemma; a consensus of opinion was only reached after discussion between the patient and a multidisciplinary team, including the operative risk from both maternal and fetal aspects. We decided to remove the stone in this case and surgery was planned to occur during the 2nd trimester of pregnancy in order to avoid anesthetic risk, spontaneous abortion, and preterm contraction.

ESWL and PCNL are absolutely contraindicated for pregnancy and were therefore not possible for this patient. Previous literature claimed that semi-rigid URS in pregnancy was safe with an overall complication rate of $4-8.3 \%$, which is comparable with the rate for nonpregnant women. However, this technique is not suitable for the removal of kidney stones Flexible ureterorenoscopy appeared to represent the best option for our patient. We planned to use a UAS while performing RIRS so that we could reduce intrarenal pressure and reduce the risk of ureteric wall injury from repeated stone extraction. ${ }^{2,4}$ We carried out three operations with a UAS and did not observe any ureteric injuries. UAS was safe and easily applied without any forceful insertion, probably due to the ureteric dilatation caused by progesterone. However, the 2nd and 3rd procedures were both pre-stented.

Fluoroscopy should be avoided during pregnancy although there is good evidence that ultrasonography can be an efficient technique when assessing the positioning of guidewires. The lithotripter device that we chose to use in this surgery was the Ho-YAG laser; this was due to its flexibility, efficacy, and safety profile. ${ }^{3}$

Previous literature reports that the stone-free rate of RIRS is only $35-58 \%$ when applied to kidney stones that are approximately $3 \mathrm{~cm}$ in diameter. However, the use of auxiliary and staged procedures can increase the stone-free status to $89.3 \%$ of cases in an average of 1.6 procedures. ${ }^{4}$ Our patient received 3 sessions of RIRS and 2 changes of ureteric stent; however, the kidney stone was successfully removed in its entirety.

The independent risk factors for urinary tract infection after RIRS are staghorn stones, operative time, and a history of urinary tract infection. Although we limited the operative time to 60 minutes, with low flow irrigation, gentle maneuvering, and the use of appropriate prophylactic antibiotics, the patient still developed postoperative fever after the 1st stage of RIRS. We realized that a sterile urine culture prior to surgery may not exclude postoperative infections because bacteria may also be present on the stone itself. However, after the 2nd and 3rd stages of RIRS, the patient did not develop fever. The only difference between the 1st stage and the 2nd and 3rd stages was related to the ureteral stent. In the 1st stage, the urine in the kidney had been drained by an ureteral stent. The larger UAS used in the 2 nd and 3 rd procedures may have reduced intrarenal pressure more effectively than the stent used in the 1st stage of RIRS.

\section{Conclusion}

We successfully removed a large kidney stone from a pregnant patient using an ureteral access sheath and RIRS without fluoroscopy. There were no complications indicating that this procedure can be carried out safely during pregnancy. Postoperative infection should be considered, and any obstruction should be drained before the operation. If dealing with large stones, it is important to consider staged surgical intervention.

\section{Ethical Approval}

The publication of this study was approved by the Ethics Committee of the Faculty of Medicine Siriraj 
hospital, Mahidol University, Thailand. The patient provided informed and signed consent to have the case details and any accompanying images published.

\section{Acknowledgment}

There was no research support/fund in this study.

\section{Disclosure}

None of the authors have any conflicts of interest to report for this work.

\section{References}

1. Valovska M, Pais V. Contemporary best practice urolithiasis in pregnancy. Ther Adv Urol. 2018;10(4):127-138. doi:10.1177/1756 287218754765

2. Rivera M, Viers B, Cockerill P, et al. Pre- and postoperative predictors of infection-related complications in patients undergoing percutaneous nephrolithotomy. J Endourol. 2016;30(9):982-986. doi:10.1089/end. 2016.0191

3. Akpinar H, Tüfek İ, Alici B, Kural A. Ureteroscopy and holmium laser lithotripsy in pregnancy: stents must be used postoperatively. $J$ Endourol. 2006;20(2):107-110. doi:10.1089/end.2006.20.107

4. Breda A, Angerri O. Retrograde intrarenal surgery for kidney stones larger than $2.5 \mathrm{~cm}$. Curr Opin Urol. 2014;24(2):179-183. doi:10.1097/ MOU.0000000000000030

\section{Publish your work in this journal}

Research and Reports in Urology is an international, peer-reviewed, open access journal publishing original research, reports, editorials, reviews and commentaries on all aspects of adult and pediatric urology in the clinic and laboratory including the following topics: Pathology, pathophysiology of urological disease; Investigation and treatment of urological disease; Pharmacology of drugs used for the treatment of urological disease. The manuscript management system is completely online and includes a very quick and fair peer-review system, which is all easy to use. Visit http://www.dovepress.com/ testimonials.php to read real quotes from published authors. 